J. Nonlinear Var. Anal. 4 (2020), No. 1, pp. 153-157

Available online at http://jnva.biemdas.com

https://doi.org/10.23952/jnva.4.2020.1.11

\title{
A GLOBAL APPROXIMATE CONTRACTION MAPPING PRINCIPLE IN NON-COMPLETE METRIC SPACES
}

\author{
M. AIT MANSOUR ${ }^{1, *}$, M.A. BAHRAOUI ${ }^{2}$, A. EL BEKKALI ${ }^{2}$ \\ ${ }^{1}$ Département de Physiques, LPFAS, Faculté Poly-disciplinaire, Safi, Université Cadi Ayyad, Morocco \\ ${ }^{2}$ Département de Mathématiques, Faculté des Sciences et Techniques, Tanger, Université Abdelmalek Essaadi, Morocco
}

\begin{abstract}
In this paper, we present a result which simultaneously provides the existence of approximate fixed points for a setvalued contraction mapping in a not necessarily complete metric space, and estimate the distance from a point to the set of the approximate fixed points of the underlying map. As an application, we give a new characterization of Lipschitzian set-valued mappings.
\end{abstract}

Keywords. Fixed point; Approximate fixed point; Noncomplete metric space; Contraction mapping.

\section{INTRODUCTION AND PRELIMINARIES}

The Lipschitzian continuity of functions is very important in many areas of analysis. In particular, it plays a fundamental role in variational analysis. It has been extended to set-valued maps with the use of the Hausdorff distance and it now leads to a quantitative continuity mode for such maps which serves in particular evaluating rates of changes of their images. For detailed expositions on this concept and its important uses, the reader is refereed for example to the books [1, 2]. The typical case when the Lipschitz constant of a set-valued map is in the interval $(0,1)$ covers the class of contractions, which find wide fields of applications, such as, fixed point theory, quantitative stability of systems of variational inequalities, approximations in numerical treatments and so forth.

In this paper, we give a new characterization of Lipschitz continuity of set-valued maps by using approximate fixed points in the setting of a not necessarily complete metric space. Let us fix our notations and basic tools. Throughout the paper, $(X, d)$ is a metric space and $\mathscr{C}(X)$ is the set of nonempty and closed subsets of $X$. The Hausdorff distance between two subsets $A$ and $B$ of $X$ is given by

$$
D(A, B)=\max \left\{\sup _{a \in A} d(a, B), \sup _{b \in B} d(b, A)\right\} .
$$

Let $(Y, \delta)$ be an another metric space and $\Delta$ the Hausdorff distance associated to $\delta$. A set-valued mapping $\Phi: X \rightrightarrows Y$ is said to be Lipschitzian if there exists a constant $\kappa>0$ such that

$$
\Delta\left(\Phi(x), \Phi\left(x^{\prime}\right)\right) \leqslant \kappa d\left(x, x^{\prime}\right)
$$

\footnotetext{
${ }^{*}$ Corresponding author.

E-mail addresses: ait.mansour.mohamed@gmail.com (M. Ait Mansour), mbahraoui@ hotmail.com (M.A. Bahraoui), adhamcpge@gmail.com (A. El Bekkali).

Received December 16, 2019; Accepted March 1, 2020.
}

(C)2020 Journal of Nonlinear and Variational Analysis 
for all $x, x^{\prime} \in X$. The constant $\kappa$ is called Lipschitz constant of $\Phi$. If $\kappa<1, \Phi$ is said to be a set-valued contraction. A useful characterization of Lipschitzian set-valued mappings is given in the following result.

Theorem 1.1 ([1, Proposition 3C.1]). Let $(X, d)$ and $(Y, \delta)$ be two metric spaces. A set-valued mapping $\Phi: X \rightrightarrows Y$ is Lipschitzian with constant $\kappa$ if and only if

$$
d(y, \Phi(x)) \leqslant \kappa d\left(x, \Phi^{-1}(y)\right), \quad \forall(x, y) \in X \times Y .
$$

A point $x \in X$ is said to be a fixed point of a set-valued mapping $\Phi: X \rightrightarrows X$ if $x \in \Phi(x)$. The set of fixed points of $\Phi$ is denoted by $\operatorname{Fix}(\Phi)$ in this paper. It follows from [1, Theorem 5I.3] that the distance from a point $x$ to the set of fixed points of a set-valued contraction mapping $\Phi$ is bounded by a constant times the distance from $x$ to $\Phi(x)$.

Precisely, we have the following result.

Theorem 1.2. Let $(X, d)$ be a complete metric space, and let $\Phi: X \rightarrow \mathscr{C}(X)$ be a set-valued contraction mapping with Lipschitz constant $\kappa$. Then, for every $x \in X$,

$$
d(x, \operatorname{Fix}(\Phi)) \leqslant \frac{1}{1-\kappa} d(x, \Phi(x)) .
$$

Let $\varepsilon>0$. A point $x \in X$ is said to be $\varepsilon$-fixed point of a set-valued mapping $\Phi: X \rightrightarrows X$ if $d(x, \Phi(x)) \leqslant \varepsilon$. The set of $\varepsilon$-fixed points of $\Phi$ is denoted by $\varepsilon$-Fix $(\Phi)$ in this paper.

Our goal in this work is to give an estimate of the distance from a point to the set of $\varepsilon$-fixed points of a set-valued contraction mapping, which is similar with the one given in Theorem 1.2 without any completeness assumption. As a consequence, we obtain a new characterization of Lipschitzian set-valued mappings.

\section{MAIN RESULTS}

We now present our first result below, Theorem 2.1, which provides an estimate of the distance from a point to the set of $\varepsilon$-fixed points of a set-valued contraction. Our proof is based on [4, Theorem 1.2].

Theorem 2.1. Let $(X, d)$ be a metric space, and let $\Phi: X \rightrightarrows X$ be a set-valued contraction with Lipschitz constant $\kappa$. Then, for every $\varepsilon>0, \varepsilon-\operatorname{Fix}(\Phi)$ is nonempty and

$$
d(x, \varepsilon-\operatorname{Fix}(\Phi)) \leqslant \frac{1}{1-\kappa} d(x, \Phi(x)), \quad \forall x \in X .
$$

Proof. Fix some $\varepsilon>0$. From the proof of [4, Theorem 1.2], there exist a complete metric space $(Y, \delta)$, an isometry $i: X \rightarrow Y$ such that $i(X)$ is dense in $Y$, and a contraction mapping $\Psi: Y \rightarrow \mathscr{C}(Y)$ with Lipschitz constant $\kappa$ such that, for every $x \in X$,

$$
\Psi(i(x))=\overline{i(\Phi(x))}:=\overline{\{i(x): x \in \Phi(x)\}},
$$

where $\overline{i(\Phi(x))}$ is the closure of $i(\Phi(x))$ in $Y$.

Let $x \in X$. Since $Y$ is complete, it follows from Theorem 1.2 that

$$
\delta(i(x), \operatorname{Fix}(\Psi)) \leqslant \frac{1}{1-\kappa} \delta(i(x), \Psi(i(x))) .
$$


Let $\alpha \in(0, \varepsilon)$. There exists $y \in \operatorname{Fix}(\Psi)$ such that

$$
\delta(i(x), y)<\delta(i(x), \operatorname{Fix}(\Psi))+\frac{\alpha}{2} .
$$

On the other hand, since $i(X)$ is dense in $Y$, we have that there exists $x_{0} \in X$ such that

$$
\delta\left(i\left(x_{0}\right), y\right) \leqslant \frac{\alpha}{2} .
$$

Thus,

$$
\begin{aligned}
d\left(x_{0}, \Phi\left(x_{0}\right)\right) & =\delta\left(i\left(x_{0}\right), \overline{i\left(\Phi\left(x_{0}\right)\right)}\right) \\
& =\delta\left(i\left(x_{0}\right), \Psi\left(i\left(x_{0}\right)\right)\right) \\
& \leqslant \delta\left(i\left(x_{0}\right), y\right)+\delta\left(y, \Psi\left(i\left(x_{0}\right)\right)\right) \\
& \leqslant \delta\left(i\left(x_{0}\right), y\right)+\Delta\left(\Psi(y), \Psi\left(i\left(x_{0}\right)\right)\right) \\
& \leqslant \delta\left(i\left(x_{0}\right), y\right)+\kappa \delta\left(y, i\left(x_{0}\right)\right) \\
& =(\kappa+1) \delta\left(y, i\left(x_{0}\right)\right) \\
& \leqslant \alpha .
\end{aligned}
$$

Accordingly, $x_{0} \in \alpha-\operatorname{Fix}(\Phi) \subset \varepsilon-\operatorname{Fix}(\Phi)$. Hence,

$$
\begin{aligned}
d(x, \mathcal{\varepsilon}-\operatorname{Fix}(\Phi)) & \leqslant d\left(x, x_{0}\right)=\delta\left(i(x), i\left(x_{0}\right)\right) \\
& \leqslant \delta(i(x), y)+\delta\left(y, i\left(x_{0}\right)\right) \\
& \leqslant \delta(i(x), \operatorname{Fix}(\Psi))+\alpha \\
& \leqslant \frac{1}{1-\kappa} \delta(i(x), \Psi(i(x)))+\alpha \\
& \leqslant \frac{1}{1-\kappa} \delta(i(x), \overline{i(\Phi(x))})+\alpha \\
& \leqslant \frac{1}{1-\kappa} d(x, \Phi(x))+\alpha .
\end{aligned}
$$

By passing to the limit when $\alpha$ goes to 0 , we obtain the estimate in theorem. This completes the proof.

Remark 2.1. We remark that Theorem 2.1 covers [4, Theorem 1.2], which claims the existence of $\varepsilon$-fixed points, for every $\varepsilon>0$, of set-valued contractions in any metric space.

Under some conditions of completeness and closedness, it was established in [3, Theorem 6] a characterization of Lipschitzian set-valued mappings by using fixed points. Our aim here is to give, without any condition of completeness or closedness, a similar characterization of Lipschitzian set-valued mappings by involving approximate fixed points instead of exact ones. To do this, we will need the following elementary result whose proof is straight and we omit it here.

Proposition 2.1. Let $F: X \rightrightarrows Y$ be a Lipschitzian set-valued mapping with Lipschitz constant $\kappa$ and let $F: Y \rightrightarrows Z$ be a Lipschitzian set-valued mapping with Lipschitz constant $\mu$. If $G \circ F: X \rightrightarrows Z$ is defined by $G \circ F(x)=\cup_{y \in F(x)} G(y)$ for all $x \in X$, then $G \circ F$ is a Lipschitzian set-valued mapping with Lipschitz constant $\kappa \mu$.

Now, we state our characterization of Lipschitzian set-valued mappings. 
Theorem 2.2. Let $(X, d)$ and $(Y, \delta)$ be metric spaces. Let $\kappa$ and $\mu$ be nonnegative constants such that $\kappa \mu<1$. Let $F: Y \rightrightarrows X$ be a set-valued mapping. Then the following assertions are equivalent:

(i) $F$ is Lipschitizian with constant $\kappa$.

(ii) For any set-valued mapping $G: X \rightrightarrows Y$, which is Lipschitzian with constant $\mu$ and for every $\varepsilon>0$, one has

$$
d(x, \varepsilon-\operatorname{Fix}(F \circ G)) \leqslant \frac{\kappa}{1-\kappa \mu} d\left(F^{-1}(x), G(x)\right), \quad \text { for all } x \in X .
$$

Proof. Assume that $F$ is Lipschitizian with constant $\kappa$. Let $G: X \rightrightarrows Y$ be a Lipschitzian set-valued mapping with Lipschitz constant $\mu$, and let $x \in X$ and $\varepsilon>0$. It follows from Proposition 2.1 that $F \circ G$ is Lipschitzian with constant $\kappa \mu$. Since $\kappa \mu<1$, we can apply Theorem 2.1 with $\Phi=F \circ G$. In this way, we obtain

$$
d(x, \varepsilon-\operatorname{Fix}(F \circ G)) \leqslant \frac{1}{1-\kappa \mu} d(x, F \circ G(x)) .
$$

Since $F$ is Lipschitzian with constant $\kappa$, it follows from Theorem 1.1 that

$$
d(x, F(y)) \leqslant \kappa d\left(y, F^{-1}(x)\right) \text { for all } y \in Y .
$$

By taking the infimum in (2.3) with respect to $y \in G(x)$, we obtain

$$
d(x, F \circ G(x)) \leqslant \kappa d\left(G(x), F^{-1}(x)\right) .
$$

Hence, combining (2.2) and (2.4), we obtain the required estimate

$$
d(x, \varepsilon-\operatorname{Fix}(F \circ G)) \leqslant \frac{\kappa}{1-\kappa \mu} d\left(F^{-1}(x), G(x)\right) .
$$

Now, we suppose that (ii) holds. Let $y \in Y$ and consider the constant mapping $G: X \rightrightarrows Y$ defined by $G(x)=y$ for all $x \in X$. Clearly, $G$ is Lipschitzian with constant $\mu=0$. Then, from (2.1), it results

$$
d(x, \varepsilon-\operatorname{Fix}(F \circ G)) \leqslant \kappa d\left(y, F^{-1}(x)\right) .
$$

If $d\left(y, F^{-1}(x)\right)=+\infty$, we automatically obtain

$$
d\left(y, F(x) \leqslant \kappa d\left(y, F^{-1}(x)\right) .\right.
$$

Next, we assume that

$$
d\left(y, F^{-1}(x)\right)<+\infty
$$

Thanks to (2.6), we have

$$
d(x, \varepsilon-\operatorname{Fix}(F \circ G))<\kappa d\left(y, F^{-1}(x)\right)+\varepsilon .
$$

Thus, there exists $u \in \varepsilon$-Fix $(F \circ G)$ such that

$$
d(x, u)<\kappa d\left(y, F^{-1}(x)\right)+\varepsilon .
$$

On the other hand, we also have

$$
d(u, F(y))=d(u, F \circ G(u)) \leqslant \varepsilon .
$$

Therefore, by combining (2.7) and (2.8), we obtain

$$
d(x, F(y)) \leqslant d(x, u)+d(u, F(y)) \leqslant \kappa d\left(y, F^{-1}(x)\right)+2 \varepsilon .
$$

By converging $\varepsilon$ to 0 in (2.9) we get

$$
d\left(x, F(y) \leqslant \kappa d\left(y, F^{-1}(x)\right) .\right.
$$


According to Theorem 1.1, this latter means that $F$ is Lipschitzian with constant $\kappa$. This completes the proof.

\section{Acknowledgements}

The authors thank the anonymous referee for her/his valuable comments on the first version of this paper.

\section{REFERENCES}

[1] A. L. Dontchev, R. T. Rockafellar, Implicit Functions and Solution Mappings, Springer, New York, 2014.

[2] R.T. Rockafellar, R.J.-B. Wets, Variational Analysis, Springer, Berlin, 1998.

[3] S. Adly, A. L. Dontchev, M. Théra, On one-sided Lipschitz stability of set-valued contractions, Numer. Funct. Anal. Optim. 35 (2014), 837-850.

[4] M. Ait Mansour, M. A. Bahraoui, A. El Bekkali, Approximate fixed points via completion, Le Matematiche 74 (2019), $411-415$. 